\title{
ON THE ASYMPTOTIC GEOMETRY OF AREA-PRESERVING MAPS
}

\author{
Leonid Polterovich and Karl Friedrich Siburg \\ We dedicate this paper to the memory of Jürgen Moser
}

\section{Statement and discussion of the results}

Let $M$ be an open connected oriented 2-manifold endowed with an area form $\omega$. We assume that the total area of $M$ with respect to $\omega$ is infinite, i.e., $\int_{M} \omega=\infty$. Consider the group $\operatorname{Ham}_{c}(M, \omega)$ of Hamiltonian diffeomorphisms of $M$ consisting of all time-1-maps of time-periodic compactly supported Hamiltonians $H: \mathbb{S}^{1} \times M \rightarrow \mathbb{R}$. We write $\varphi_{H}^{t}$ for the Hamiltonian flow generated by $H$.

In this paper, we study the asymptotic behaviour of one-parameter subgroups of $\operatorname{Ham}_{c}(M, \omega)$ with respect to Hofer's metric $d$ where ${ }^{1}$

$d(\mathrm{id}, \varphi)=\inf \left\{\int_{0}^{1} \max H_{t}-\min H_{t} d t \mid H\right.$ has compact support and $\left.\varphi_{H}^{1}=\varphi\right\}$.

Let $\mathcal{A}$ denote the Lie algebra of $\operatorname{Ham}_{c}(M, \omega)$; it consists of all compactly supported time-independent Hamiltonians on $M$. Given $H \in \mathcal{A}$, we are interested in the growth of the function $r_{H}:[0, \infty) \rightarrow[0, \infty)$ defined by

$$
r_{H}(t)=d\left(\mathrm{id}, \varphi_{H}^{t}\right) \text {. }
$$

By the triangle inequality for Hofer's metric we know that $r_{H}$ is subadditive, i.e.,

$$
r_{H}(t+s) \leq r_{H}(t)+r_{H}(s)
$$

Therefore the limit

$$
\mu(H)=\lim _{t \rightarrow \infty} \frac{r_{H}(t)}{t}
$$

is well defined. This quantity — which is called the asymptotic non-minimality of the subgroup generated by $H$ - was introduced in [BP]; see also [Po2] for further discussion.

In particular, we see that $r_{H}$ grows at most linearly in $t$. On the other hand, if $(M, \omega)$ is the standard Euclidean plane then a theorem by Sikorav ([Si, HZ]; see also Proposition 3.2 below) states that $r_{H}$ is bounded by a constant, and this constant depends only on the diameter of the $\operatorname{support} \operatorname{supp}(H)$ of $H$.

Received May 20, 1999.

${ }^{1}$ We refer the reader to [HZ, MS, Po2] for an introduction to Hofer's geometry. 
In the present note, we show that for open surfaces of infinite area the function $r_{H}$ is either bounded or behaves asymptotically linear. In order to formulate our main result we recall that a subset $Z \subset M$ is called contractible in $M$ if the inclusion $Z \hookrightarrow M$ is homotopic to the constant map which maps the whole $Z$ to a point in $M$.

Theorem 1.1 (Dichotomy Theorem). Let $(M, \omega)$ be an open surface of infinite area and $H: M \rightarrow \mathbb{R}$ a compactly supported Hamiltonian. Then the following dichotomy holds:

- If $\{H \neq 0\}$ is contractible in $M$ then the function $r_{H}$ is bounded; in particular, $\mu(H)=0$.

- If $\{H \neq 0\}$ is not contractible in $M$ then the function $r_{H}$ grows asymptotically linear, i.e., $\mu(H)>0$.

As a consequence, we get the following criterion for the boundedness of the function $r_{H}$ in more dynamical terms; see Section 2 for the proof.

Corollary 1.2. The function $r_{H}$ is bounded if and only if all periodic orbits of the Hamiltonian flow $\varphi_{H}^{t}$ are contractible.

In fact, it is even possible to calculate the precise value of $\mu(H)$ as the difference of two distinguished critical values of $H$. In particular, one gets examples of one-parameter subgroups whose asymptotic non-minimality lies strictly between 0 and $\max H-\min H$ and can be calculated precisely. As far as we know, this is the first series of examples of that type.

Assume that $M \neq \mathbb{R}^{2}$. Then $\pi_{1}(M)$ is nontrivial. Let $\mathcal{L}$ be the set of all embedded non-contractible circles in $M$. Then we define

$$
c_{+}(H)=\sup _{L \in \mathcal{L}} \min _{x \in L} H(x), \quad c_{-}(H)=\inf _{L \in \mathcal{L}} \max _{x \in L} H(x) .
$$

Since $H$ is compactly supported, it follows that $c_{+}(H) \geq 0$ and $c_{-}(H) \leq 0$ (see Proposition 2.4). Moreover, one can easily check (see Proposition 2.3) that $c_{+}(H)$ and $c_{-}(H)$ are critical values of $H$.

Theorem 1.3. The following equality holds:

$$
\mu(H)=c_{+}(H)-c_{-}(H) .
$$

We give a short outline of the proof of the two theorems. If $\{H \neq 0\}$ is contractible then a version of the abovementioned theorem by Sikorav shows that $r_{H}$ is bounded by a constant depending only on the "size" of $\operatorname{supp}(H)$. Thus we get the first statement of Theorem 1.1 (see Section 3 below). An elementary argument (see Proposition 2.5) shows that $c_{-}(H)=c_{+}(H)=0$ if and only if $\{H \neq 0\}$ is contractible in $M$. Thus, the second statement of Theorem 1.1 follows from Theorem 1.3. Theorem 1.3 consists of two parts. The inequality $\mu(H) \geq c_{+}(H)-c_{-}(H)$ follows from a Lagrangian intersection result as in [Po1] (see Section 4). The proof of the reversed inequality uses a trick, namely a decomposition of $\varphi_{H}^{t}$ into two commuting flows:

$$
\varphi_{H}^{t}=\Phi^{t} \circ \Psi^{t}=\Psi^{t} \circ \Phi^{t},
$$


where $\Phi^{t}$ has contractible support. Hence, Sikorav's theorem yields that $\Phi^{t}$ can be neglected in the calculation of $\mu(H)$ (see Section 3). Let us emphasize that in order to apply Sikorav's argument we need that $M$ has infinite area.

Intuitively, the two distinguished critical values $c_{ \pm}(H)$ correspond to the first homotopically nontrivial separatrices of $H$. More precisely, $c_{+}(H)$ is the infimum of energy values $E$ such that the superlevel set $H^{-1}([E, \infty))$ is contractible, and $c_{-}(H)$ is the supremum of $E$ with contractible sublevel set $H^{-1}((-\infty, E])$. Consequently, the asymptotic geometric behaviour of $\varphi_{H}^{t}$ depends only on the topology of the level sets of $H$.

When $M$ is the cylinder, the lower bound on the asymptotic non-minimality $\mu(H)$ in terms of the energy levels which carry non-contractible circles was known [Po2, 9.B]. Theorem 1.3 above shows that this bound is sharp!

Concerning the function $r_{H}(t)$, we obtain the following picture. As long as there are no non-constant periodic solutions we have

$$
r_{H}(t)=(\max H-\min H) t,
$$

see [LM, II, Cor. 1.10]. For large $t$, however,

$$
r_{H}(t) \sim\left(c_{+}(H)-c_{-}(H)\right) t .
$$

Consequently, if $\max H>c_{+}(H)$ or $c_{-}(H)>\min H$ there must be a "phase transition" in the behaviour of $r_{H}(t)$ from small $t$ to large $t$.

Let us conclude with a couple of open problems. First of all, it is not clear if the Dichotomy Theorem holds true for surfaces of finite area, or, even more ambitious, for higher-dimensional symplectic manifolds. We also do not know how to deal with cyclic subgroups of $\operatorname{Ham}_{c}(M, \omega)$, consisting of time-1-maps of time-dependent Hamiltonians periodic in time. The reason is the lack of an integral of motion which is essential for our arguments. Finally, it would be interesting to have a dynamical interpretation of the abovementioned change in the behaviour of $r_{H}(t)$.

\section{Some properties of $c_{ \pm}(H)$}

In this section we sum up some useful elementary properties of $c_{ \pm}(H)$, deduce the second statement of Theorem 1.1 from Theorem 1.3 and prove Corollary 1.2.

We start with some auxiliary facts and notions from the topology of open surfaces.

Proposition 2.1. An open subset $Z \subset M$ is contractible in $M$ if and only if every embedded circle which lies in $Z$ is contractible in $M$.

Proof. Assume without loss of generality that $Z$ is connected. Since an open surface is a $K(\pi, 1)$-space, the inclusion $Z \hookrightarrow M$ is homotopic to a point if and only if the homomorphism $\pi_{1}(Z) \rightarrow \pi_{1}(M)$ is trivial [Sp, 8.1.11]. But $Z$ is also an open surface. Representing $Z$ as the union of an increasing chain of compact surfaces with boundary we see that there exist countably many embedded circles which generate $\pi_{1}(Z)$. Hence, $Z$ is contractible if and only if all these generating circles are contractible. 
Every contractible embedded circle $\gamma$ on $M$ bounds a unique closed disc which we denote $D(\gamma)$. For an arbitrary subset $X \subset M$ set $^{2}$

$$
\operatorname{hull}(X)=\operatorname{cl}\left(\cup_{\gamma} D(\gamma)\right),
$$

where $\gamma$ runs over all contractible embedded circles which are contained in $X$.

When $N \subset M$ is a compact submanifold with boundary it is not hard to show that its hull coincides with the union of $N$ with all discs $D(\gamma)$, where $\gamma$ stands for a contractible boundary circle of $N$. As an immediate consequence we get that the hull of a compact subset of $M$ is compact. Indeed, each compact subset is contained in some compact submanifold with boundary. In what follows, we need a finer description of $\operatorname{hull}(N)$ in the case when $N \subset M$ is a compact submanifold with contractible boundary.

Proposition 2.2. Let $N \subset M$ be a 2-dimensional compact submanifold with boundary. Assume that $\partial N$ is contractible in $M$. Then hull $(N)$ is the union of a finite number of pairwise disjoint closed discs whose boundaries are connected components of $\partial N$. In particular, $N$ is contractible in $M$.

Proof. It suffices to show that there exist pairwise disjoint closed embedded discs $D_{1}, \ldots, D_{k} \subset M$ such that $\partial D_{j}$ is a component of $\partial N$ and $N \subset \cup_{j} D_{j}$. Since $N$ is compact it has only finitely many connected components which we denote by $N_{i}$; let $\gamma_{i j}$ denote the boundary components of $N_{i}$. Now fix some $i$. Since $M$ is open and $\partial N$ contractible, at least one of the discs $D\left(\gamma_{i j}\right)$ intersects the interior of $N_{i}$. Denote this disc by $D_{i}$.

We claim that $D_{i}$ contains $N_{i}$. Indeed, pick any point $x \in \operatorname{int}\left(D_{i} \cap N_{i}\right)$, and assume on the contrary that there exists a point $y \in \operatorname{int}\left(N_{i}\right) \backslash D_{i}$. Since $N_{i}$ is connected, there exists a path $\alpha$ in the interior of $N_{i}$ which joins $x$ and $y$. But, since $x$ lies inside $D_{i}$ and $y$ outside $D_{i}, \alpha$ must intersect $\partial D_{i} \subset \partial N$ which is impossible. This contradiction proves the claim.

Notice that for $i \neq j$ either $D_{i}$ and $D_{j}$ are disjoint, or one contains the other. So choose from the set $\left\{D_{1}, \ldots, D_{p}\right\}$ those discs which are maximal with respect to inclusion. This family of discs satisfies all the above requirements.

Let us return now to the quantities $c_{ \pm}$. As before we assume that $M \neq \mathbb{R}^{2}$. The next proposition is quite standard in the calculus of variations and could be formulated, for instance, in the setting of the Minimax Lemma in [HZ, Sect. 3.2]. For the convenience of the reader, we give a slightly more direct proof here.

Proposition 2.3. $c_{ \pm}(H)$ are critical values of $H$.

Proof. Assume on the contrary that $c_{+}(H)$ is a regular value of $H$. Then there exists a segment $\left[c, c^{\prime}\right]$ which consists of regular values of $H$ and such that $c<c_{+}(H)<c^{\prime}$. By definition of $c_{+}(H)$, the set $\{H \geq c\}$ contains a noncontractible circle. Since the gradient flow of $H$ takes $\{H \geq c\}$ into $\left\{H \geq c^{\prime}\right\}$, we conclude $\left\{H \geq c^{\prime}\right\}$ also contains a non-contractible circle. Hence $c_{+}(H) \geq c^{\prime}$, in contradiction to the choice of $c^{\prime}$.

\footnotetext{
${ }^{2}$ We write $\operatorname{cl}(Z)$ and $\operatorname{int}(Z)$ for the closure and the interior of a subset $Z$, respectively.
} 
The proof for $c_{-}(H)$ is analogous.

Proposition 2.4. $c_{+}(H) \geq 0$ and $c_{-}(H) \leq 0$.

Proof. It suffices to show that the set $M \backslash \operatorname{supp}(H)$ contains an embedded noncontractible circle. Write $M=\cup_{i} N_{i}$, where $N_{i}$ are compact surfaces with boundary such that $\operatorname{supp}(H) \subset \operatorname{int}\left(N_{1}\right)$ and $N_{i} \subset \operatorname{int}\left(N_{i+1}\right)$ for all $i \geq 1$.

If some boundary component of some $N_{i}$ is non-contractible in $M$ we are done. Assume therefore that all of them are contractible. Then Proposition 2.2 implies that all $N_{i}$ are contractible in $M$. We conclude that $\pi_{1}(M)=0$, i.e., $M=\mathbb{R}^{2}$, in contradiction to our standing assumption.

Proposition 2.5. $c_{-}(H)=c_{+}(H)=0$ if and only if $\{H \neq 0\}$ is contractible in $M$.

Proof. We are going to apply Proposition 2.1 with $Z=Z_{H}=\{H \neq 0\}$.

If $Z_{H}$ is not contractible in $M$ then it contains a curve from $\mathcal{L}$. This curve lies either in $\{H>0\}$, which implies $c_{+}(H)>0$, or in $\{H<0\}$, in which case $c_{-}(H)<0$.

Suppose now that $Z_{H}$ is contractible in $M$. Then $Z_{H}$ cannot contain a curve from $\mathcal{L}$. This means that every curve from $\mathcal{L}$ intersects the set $\{H=0\}$, so $c_{+}(H) \leq 0$ and $c_{-}(H) \geq 0$. But, as we have seen in Proposition 2.4, $c_{+}(H)$ is non-negative and $c_{-}(H)$ is non-positive. Therefore $c_{-}(H)=c_{+}(H)=0$.

As a consequence we see that the second statement of Theorem 1.1 follows from Theorem 1.3.

We conclude this section with the proof of Corollary 1.2 stating that the function $r_{H}(t)=d\left(\mathrm{id}, \varphi_{H}^{t}\right)$ is bounded if and only if all periodic orbits of $\varphi_{H}^{t}$ are contractible.

Proof of Corollary 1.2. In view of Theorem 1.1, we have to show that the set $\{H \neq 0\}$ is contractible if and only if all periodic orbits are contractible.

Assume first that some energy level $\{H=c\}$ contains a non-contractible closed orbit $L$. Note that there are no critical points of $H$ on $L$. Thus for every $c^{\prime} \in \mathbb{R}$ close enough to $c$, also the level $\left\{H=c^{\prime}\right\}$ contains a non-contractible embedded circle. Choosing $c^{\prime} \neq 0$ we get that the set $\{H \neq 0\}$ contains a noncontractible embedded circle. Therefore this set is not contractible in $M$ in view of Proposition 2.1.

Assume now that every closed orbit of the Hamiltonian flow generated by $H$ is contractible. Arguing by contradiction, we suppose the set $\{H \neq 0\}$ is not contractible in $M$. In view of Proposition 2.1, there exists a non-contractible embedded circle $L$ on which $H$ does not vanish. Assume without loss of generality that $\left.H\right|_{L}>0$. Choose a regular value $c$ of $H$ such that $0<c<\min _{L} H$, and pick any $c^{\prime} \in\left(c, \min _{L} H\right)$. Note that the open set $N^{\prime}=\left\{H>c^{\prime}\right\}$ is not contractible in $M$ since it contains $L$ (apply again Proposition 2.1). We want to show that this leads to a contradiction. For this, consider the set $N=\{H \geq c\}$ which is a compact submanifold of $M$ whose boundary consists of closed orbits of the 
flow. By our assumption, this boundary is contractible in $M$, so Proposition 2.2 implies that $N$ is contractible in $M$. But then also $N^{\prime} \subset N$ is contractible in $M$, and we arrive at a contradiction. Therefore $\{H \neq 0\}$ is contractible in $M$. This completes the proof.

\section{Decomposing Hamiltonian flows}

In this section we prove the following result.

Theorem 3.1. The following inequality holds:

$$
\mu(H) \leq c_{+}(H)-c_{-}(H) .
$$

Moreover, if $c_{-}(H)=c_{+}(H)=0$ then $r_{H}$ is bounded.

Together with Propositions 2.4 and 2.5 this implies the first statement of Theorem 1.1.

An essential ingredient of our approach is the following version of Sikorav's theorem [Si]; see also [HZ, Sect. 5.6]. If $X \subset(M, \omega)$, we write area $(X)$ for $\int_{X} \omega$.

Proposition 3.2. Let $X \subset M$ be a finite union of pairwise disjoint closed discs, and let $F \in \mathcal{A}$ be a Hamiltonian function on $M$ whose support is contained in the interior of $X$. Then

$$
d\left(\mathrm{id}, \varphi_{F}^{t}\right) \leq 16 \text { area }(X)
$$

for every $t$.

Proof. When $M=\left(\mathbb{R}^{2}, d p \wedge d q\right)$ this is proved in [Si, HZ]. The case of a general open surface of infinite area can be reduced to this one as follows. Assume without loss of generality that $X \subset(M, \omega)$ consists of just one disc of area $A$. Let $D \subset\left(\mathbb{R}^{2}, d p \wedge d q\right)$ be the closed standard disc of area $A$. Since $M$ has infinite area, it is an easy consequence of the Dacorogna-Moser theorem ([DM], see also [HZ, Sect. 1.6]) that there exists a symplectic embedding

$$
i:\left(\mathbb{R}^{2}, d p \wedge d q\right) \hookrightarrow(M, \omega),
$$

such that $i(D)=X$. Clearly, $i$ induces the natural homomorphism

$$
i_{*}: \operatorname{Ham}_{c}\left(\mathbb{R}^{2}, d p \wedge d q\right) \rightarrow \operatorname{Ham}_{c}(M, \omega) .
$$

It is important to notice that $i_{*}$ does not increase the corresponding Hofer distances. Our flow $\varphi_{F}^{t}$ lies in the image of $i_{*}$, i.e., $\varphi_{F}^{t}=i_{*}\left(f_{t}\right)$ where $f_{t}$ is a one-parameter subgroup of $\operatorname{Ham}_{c}\left(\mathbb{R}^{2}, d p \wedge d q\right)$ whose Hamiltonian is supported in $\operatorname{int}(D)$. Thus, the desired inequality follows from Sikorav's original theorem since $d\left(\mathrm{id}, \varphi_{F}^{t}\right) \leq d\left(\mathrm{id}, f_{t}\right) \leq 16 \mathrm{~A}$.

Proof of Theorem 3.1. Let us decompose the flow $\varphi_{H}^{t}$ into two commuting flows as follows. Fix some $\epsilon>0$, and choose a smooth function $\rho: \mathbb{R} \rightarrow \mathbb{R}$ satisfying the following properties:

1. $\rho(s)=s$ if $c_{-}(H)-\epsilon \leq s \leq c_{+}(H)+\epsilon$

2. $\rho(s)=c_{+}(H)+2 \epsilon$ if $s \geq c_{+}(H)+3 \epsilon$ 
3. $\rho(s)=c_{-}(H)-2 \epsilon$ if $s \leq c_{-}(H)-3 \epsilon$

4. $0<\rho^{\prime}(s)<1$ if $c_{-}(H)-3 \epsilon<s<c_{-}(H)-\epsilon$ or $c_{+}(H)+\epsilon<s<c_{+}(H)+3 \epsilon$ Define the new Hamiltonians $K=\rho \circ H$ and $H_{0}=H-K$, and denote their flows by $\Psi^{t}$ and $\Phi^{t}$, respectively. Then

$$
\varphi_{H}^{t}=\Phi^{t} \circ \Psi^{t}=\Psi^{t} \circ \Phi^{t}
$$

Observe that $\operatorname{supp}\left(H_{0}\right)$ is contained in the set

$$
Z(\epsilon)=H^{-1}\left(\left(-\infty, c_{-}(H)-\epsilon\right] \cup\left[c_{+}(H)+\epsilon, \infty\right)\right) .
$$

Pick $\kappa \in(0, \epsilon)$ such that $c_{-}(H)-\kappa$ and $c_{+}(H)+\kappa$ are regular values of $H$. Then $Z(\kappa)$ is a compact 2-dimensional submanifold with contractible boundary. Denote by $X$ the hull of $Z(\kappa)$. Proposition 2.2 implies that $X$ is a finite union of pairwise disjoint closed discs. Moreover, $Z(\kappa)$ is a subset of $\operatorname{supp}(H)$, so $X$ is contained in the hull of $\operatorname{supp}(H)$. Recall that this hull is compact, so it has finite area. Combining this with Proposition 3.2 above, we conclude that there is a constant $C>0$, depending only on $\operatorname{supp}(H)$ but not on $\epsilon$, such that

$$
d\left(\mathrm{id}, \Phi^{t}\right) \leq C
$$

for every $t$.

On the other hand, $\Psi^{t}$ is generated by $K$ with $\max K-\min K=c_{+}(H)-$ $c_{-}(H)+4 \epsilon$, hence

$$
d\left(\mathrm{id}, \Psi^{t}\right) \leq t\left(c_{+}(H)-c_{-}(H)+4 \epsilon\right) .
$$

Now, the relation (1) implies that

$$
d\left(\mathrm{id}, \varphi_{H}^{t}\right) \leq d\left(\mathrm{id}, \Phi^{t}\right)+d\left(\mathrm{id}, \Psi^{t}\right)
$$

Therefore

$$
d\left(\mathrm{id}, \varphi_{H}^{t}\right) \leq C+t\left(c_{+}(H)-c_{-}(H)+4 \epsilon\right)
$$

for every $\epsilon>0$, and the inequality in Theorem 3.1 follows. Moreover, if $c_{-}(H)=$ $c_{+}(H)=0$ we get that $r_{H}(t) \leq C$ for all $t \geq 0$, and Theorem 3.1 is proven.

\section{A lower bound on $\mu$ via Lagrangian intersections}

In the present section we prove the following result.

Theorem 4.1. We have the following inequality:

$$
\mu(H) \geq c_{+}(H)-c_{-}(H) .
$$

Together with Theorem 3.1 this completes the proof of Theorem 1.3, and thus that of Theorem 1.1.

We will make use of the Lagrangian suspension construction and Lagrangian intersection theory, similar to what is done in [Po1]. It is convenient to split 
Hofer's original definition for $d$ into two parts separating the maximum and minimum. Define

$$
\begin{aligned}
& d_{+}(\mathrm{id}, \varphi)=\inf _{F}\left\{\int_{0}^{1} \max _{x} F_{t} d t \mid \varphi_{F}^{1}=\varphi\right\}, \\
& d_{-}(\mathrm{id}, \varphi)=\inf _{F}\left\{\int_{0}^{1}-\min _{x} F_{t} d t \mid \varphi_{F}^{1}=\varphi\right\},
\end{aligned}
$$

where $F: \mathbb{S}^{1} \times M \rightarrow \mathbb{R}$ runs over all compactly supported Hamiltonians generating $\varphi$. Then

$$
d(\mathrm{id}, \varphi) \geq d_{+}(\mathrm{id}, \varphi)+d_{-}(\mathrm{id}, \varphi) .
$$

Recall that we consider $\varphi=\varphi_{H}^{1}$ where $H$ is autonomous.

\section{Lemma 4.2.}

$$
\begin{aligned}
d_{+}(\mathrm{id}, \varphi) & =\inf _{F}\left\{\max _{t, x} F \mid \varphi_{F}^{1}=\varphi\right\} \\
& =\inf _{G}\left\{\max _{t, x}(H-G) \mid \varphi_{G}^{1}=\mathrm{id}\right\}, \\
d_{-}(\mathrm{id}, \varphi) & =\inf _{F}\left\{-\min _{t, x} F \mid \varphi_{F}^{1}=\varphi\right\} \\
& =\inf _{G}\left\{-\min _{t, x}(H-G) \mid \varphi_{G}^{1}=\mathrm{id}\right\} .
\end{aligned}
$$

Proof. The first equalities are proved in [Po1, §7], and the second ones in [Po1, Lemma 3.A].

Lemma 4.3. Suppose that $G: \mathbb{S}^{1} \times M \rightarrow \mathbb{R}$ is a compactly supported Hamiltonian which generates the identity: $\varphi_{G}^{1}=i d$. Let $L \subset M$ be an embedded noncontractible circle. Then there exist $t_{0} \in \mathbb{S}^{1}$ and $x_{0} \in L$ such that $G\left(t_{0}, x_{0}\right)=0$.

Proof of Theorem 4.1. Since $\varphi_{H}^{t}=\varphi_{t H}^{1}$ and $c_{ \pm}(t H)=t c_{ \pm}(H)$ for $t>0$, it suffices to show that

$$
d\left(\mathrm{id}, \varphi_{H}^{1}\right) \geq c_{+}(H)-c_{-}(H) .
$$

Fix an arbitrary $\epsilon>0$. Choose $L$ to be a non-contractible circle on $M$ such that $\left.H\right|_{L} \geq c_{+}(H)-\epsilon$. Lemmata 4.2 and 4.3 imply that $d_{+}\left(\mathrm{id}, \varphi_{H}^{1}\right) \geq c_{+}(H)-\epsilon$. Analogously, $d_{-}\left(\mathrm{id}, \varphi_{H}^{1}\right) \geq-c_{-}(H)-\epsilon$. Thus $d\left(\mathrm{id}, \varphi_{H}^{1}\right) \geq c_{+}(H)-c_{-}(H)-2 \epsilon$, for every $\epsilon>0$. Thus we get the desired inequality.

Lemma 4.3 can either be proven along the lines of Lemma 3.B in [Po1], or be reduced to that lemma; we choose the second alternative.

Let us start with the following situation. Suppose that $(V, \Omega)$ is a closed symplectic manifold of dimension $2 n$, and let $L \subset V$ be a closed Lagrangian submanifold. Consider the new symplectic manifold

$$
(P, \sigma)=\left(V \times T^{*} \mathbb{S}^{1}, \Omega \oplus d p \wedge d t\right),
$$


where $(p, t \bmod 1)$ are canonical coordinates on the cylinder $T^{*} \mathbb{S}^{1}$. Write $Z$ for the zero section $\{p=0\}$ of $T^{*} \mathbb{S}^{1}$. Assume that $L$ has the stable Lagrangian intersection property (see [Po1]) which means that

$$
\psi(L \times Z) \cap(L \times Z) \neq \emptyset
$$

for every Hamiltonian diffeomorphism $\psi$ of $(P, \sigma)$. Finally, let

$$
F: \mathbb{S}^{1} \times V \rightarrow \mathbb{R}
$$

be a time-periodic Hamiltonian function satisfying the following conditions:

(i) $F$ generates the identity, i.e., $\varphi_{F}^{1}=$ id;

(ii) $\int_{V} F(t, x) \Omega^{n}=0$ for all $t \in \mathbb{S}^{1}$;

(iii) the loop of Hamiltonian diffeomorphisms $\left\{\varphi_{F}^{t}\right\}, t \in \mathbb{S}^{1}$, is contractible in the group of all Hamiltonian diffeomorphisms of $(V, \Omega)$.

Lemma 4.4 ([Po1]). Given the above assumptions, there exist a $t_{0} \in \mathbb{S}^{1}$ and $x_{0} \in L$ such that $F\left(t_{0}, x_{0}\right)=0$.

For the sake of completeness we present the proof.

Proof. Let $A: \mathbb{S}^{1} \times V \rightarrow \mathbb{R}$ be any function which satisfies conditions (i) and (ii). Consider the so-called Lagrangian suspension

$$
\begin{aligned}
L \times \mathbb{S}^{1} & \rightarrow V \times T^{*} \mathbb{S}^{1}, \\
(x, t) & \mapsto\left(\varphi_{A}^{t}(x),-A\left(t, \varphi_{A}^{t}(x)\right), t\right) .
\end{aligned}
$$

A direct calculation shows that this is a Lagrangian embedding with respect to the symplectic form $\sigma=\Omega \oplus d p \wedge d t$ on $P=V \times T^{*} \mathbb{S}^{1}$. Denote its image by $K_{A}$. It is an easy exercise to show (see [Po3, Ch. 6] for details) that if $A(s)$ is a smooth family of functions satisfying (i) and (ii) then the corresponding Lagrangian isotopy $K_{A(s)}$ is exact, and thus extends to an ambient Hamiltonian isotopy of $P$. In particular, there exists a Hamiltonian diffeomorphism $\psi$ of $P$ such that $\psi\left(K_{A(0)}\right)=K_{A(1)}$.

The requirement that the given Hamiltonian function $F$ satisfies (iii) above guarantees that there exists a smooth family of functions $A(s)$ satisfying (i) and (ii) with $A(0) \equiv 0$ and $A(1)=F$. Since $K_{A(0)}=L \times Z$, the stable Lagrangian intersection property of $L$ yields that $K_{F} \cap(L \times Z) \neq \emptyset$. Thus there exist $t_{0} \in \mathbb{S}^{1}$ and $y_{0} \in L$ such that $\varphi_{F}^{t_{0}}\left(y_{0}\right) \in L$ and $F\left(t_{0}, \varphi_{F}^{t_{0}}\left(y_{0}\right)\right)=0$. Setting $x_{0}=\varphi_{F}^{t_{0}}\left(y_{0}\right)$ completes the proof.

Lemma 4.5. Let $(V, \Omega)$ be a closed symplectic surface, and let $L \subset V$ be a non-contractible embedded circle. Then $L$ has the stable Lagrangian intersection property.

This is a particular case of a result by Gromov [Gr, Thm. 2.3. $\left.\mathrm{B}_{3}^{\prime}\right]$; see also [Fl], and Thm. 2.3.6 of Ch. X in [AL] for a detailed exposition.

Lemma 4.6. Let $(V, \Omega)$ be a closed symplectic surface of genus at least 2 . Then $\pi_{1}(\operatorname{Ham}(V, \Omega))=0$. 
Although this fact is well known to experts, we have not been able to locate it in the literature; so we give a proof here.

Proof. Assume without loss of generality that $\int_{V} \Omega=1$. Write $\operatorname{Diff}_{0}(V)$, resp. $\operatorname{Symp}_{0}(V, \Omega)$, for the identity component of the group of all, resp. symplectic, diffeomorphisms of $V$.

Note first that the inclusion $\pi_{1}\left(\operatorname{Symp}_{0}(V, \Omega)\right) \rightarrow \pi_{1}\left(\operatorname{Diff}_{0}(V)\right)$ is an isomorphism. To see this, consider the space $X$ of all area forms on $V$ with total area 1 . Consider the mapping $\operatorname{Diff}_{0}(V) \rightarrow X$ which sends a diffeomorphism $f$ to the form $f^{*} \Omega$. One can prove a version of Moser's stability theorem [Mo] (see also [MS, Thm. 3.17]) with parameters in order to show that this mapping is a Serre fibration. Its fibre is simply $\operatorname{Symp}_{0}(V, \Omega)$, and the base $X$ is contractible. Hence the desired fact follows from the homotopy exact sequence

$$
0=\pi_{2}(X) \rightarrow \pi_{1}\left(\operatorname{Symp}_{0}(V, \Omega)\right) \rightarrow \pi_{1}\left(\operatorname{Diff}_{0}(V)\right) \rightarrow \pi_{1}(X)=0
$$

Furthermore, because the genus of $V$ is at least 2, the group $\operatorname{Diff}_{0}(V)$ is contractible [EE]. Hence $\pi_{1}\left(\operatorname{Symp}_{0}(V, \Omega)\right)=0$.

Finally, the inclusion $\pi_{1}(\operatorname{Ham}(V, \Omega)) \rightarrow \pi_{1}\left(\operatorname{Symp}_{0}(V, \Omega)\right)$ is injective [MS, 10.18 (iii).$^{3}$ Since $\pi_{1}\left(\operatorname{Symp}_{0}(V, \Omega)\right)=0$ this finishes the proof of the lemma.

Proof of Lemma 4.3. Choose a compact connected submanifold $N \subset M$ with boundary whose interior contains both $L$ and $\cup_{t} \operatorname{supp}(G(., t))$. Take two copies of $N$ and glue them together along $\partial N$. This yields a closed surface (a double of $N)$ which we denote by $V$. Let $I: V \rightarrow V$ be the reflection along $\partial N$. Extend the form $\omega$ on $N$ to an area form $\Omega$ on $V$ so that $I^{*} \Omega=-\Omega$. Furthermore, we extend the function $G(t, x)$ by 0 to $V \backslash N$ and set

$$
F(t, x)=G(t, x)-G\left(t, \varphi_{G}^{t}(I x)\right)
$$

Note that the Hamiltonian $F$ generates the flow $\varphi_{G}^{t} I \varphi_{G}^{-t} I$ and satisfies our conditions (i) and (ii) above.

Without loss of generality we can assume that $\operatorname{genus}(V) \geq 2$. Indeed, if $\operatorname{genus}(V) \leq 1$ we may add handles outside the set $\cup_{t} \operatorname{supp}(F(., t)) \cup L$, extend $\Omega$ to an area form on the handles, and set the function $F$ zero there. Applying Lemma 4.6, we see that now $F$ satisfies also the last condition (iii).

Since $L$ is non-contractible in $N$ it is also non-contractible in $V$. Lemma 4.5 yields that $L$ has the stable Lagrangian intersection property in $V$. Thus all assumptions of Lemma 4.4 are fulfilled, and we get that $F\left(t_{0}, x_{0}\right)=0$ for some $t_{0} \in \mathbb{S}^{1}$ and $x_{0} \in L$. Since $F$ and $G$ coincide on $\mathbb{S}^{1} \times N$ with $L \subset N$, we obtain $G\left(t_{0}, x_{0}\right)=0$. This completes the proof of the lemma and finishes the proof of Theorem 4.1.

\footnotetext{
${ }^{3}$ In fact, this is true for all closed symplectic manifolds.
} 


\section{Acknowledgement}

This work was done while the second author was visiting Tel Aviv University in December 1998. We thank the Hermann Minkowski Center for Geometry for the financial support.

\section{References}

[AL] M. Audin and J. Lafontaine (eds.): Holomorphic curves in symplectic geometry, Progress in Mathematics, 117, Birkhäuser Verlag, Basel, 1994

[BP] M. Bialy and L. Polterovich, Invariant tori and symplectic topology, Amer. Math. Soc. Transl. Ser. 2, 171, Amer. Math. Soc., Providence, RI, 1996, pp. 23-33.

[DM] B. Dacorogna and J. Moser, On a partial differential equation involving the Jacobian determinant, Ann. Inst. Henri Poincarè, Anal. Non Linéaire 7 (1990), 1-26.

[EE] C.J. Earle and J. Eells A fibre bundle description of Teichmüller theory, J. Diff. Geom. 3 (1969), 19-43.

[Fl] A. Floer, Cuplength estimates on Lagrangian intersections, Comm. Pure Appl. Math. 42 (1989), 335-356.

[Gr] M. Gromov, Pseudoholomorphic curves in symplectic manifolds, Invent. math. 82 (1985), 307-347.

[HZ] H. Hofer and E. Zehnder, Symplectic invariants and hamiltonian dynamics, Birkhäuser, Basel, 1994.

[LM] F. Lalonde and D. McDuff, Hofer's $L^{\infty}$-geometry: energy and stability of Hamiltonian flows I \& II, Invent. math. 122 (1995), 1-33 \& 35-69.

[MS] D. McDuff and D. Salamon, Introduction to symplectic topology, Oxford University Press, New York, 1995.

[Mo] J. Moser, On the volume elements on a manifold, Trans. Amer. Math. Soc. 120 (1965), 286-294.

[Po1] L. Polterovich, Hofer's diameter and Lagrangian intersections, Int. Math. Res. Notices 4 (1998), 217-223.

[Po2] _ Geometry on the group of Hamiltonian diffeomorphisms, Doc. Math. J. DMV, Extra Volume ICM 1998, Vol. II, 401-410.

[Po3] Geometry of the group of symplectic diffeomorphisms, Lectures in Mathematics ETH Zürich, Birkhäuser, to appear.

[Si] J.-C. Sikorav, Systèmes hamiltoniens et topologie symplectique, Università di Pisa, 1990.

[Sp] E. Spanier, Algebraic topology, McGraw-Hill, New York-Toronto, 1966.

School of Mathematical Sciences, Tel Aviv University, 69978 Tel Aviv, ISRAEL E-mail address: polterov@math.tau.ac.il

FAKultät FÜr Mathematik, Ruhr-Universität Bochum, 44780 Bochum, GERMANY E-mail address: siburg@math.ruhr-uni-bochum.de 